\title{
A MAXIMAL INEQUALITY FOR NONNEGATIVE SUB- AND SUPERMARTINGALES
}

\section{ADAM OSȨKOWSKI}

Abstract. Let $X=\left(X_{t}\right)_{t \geqslant 0}$ be a nonnegative semimartingale and $H=\left(H_{t}\right)_{t \geqslant 0}$ be a predictable process taking values in $[-1,1]$. Let $Y$ denote the stochastic integral of $H$ with respect to $X$. We show that

(i) If $X$ is a supermartingale, then

$$
\left\|\sup _{t \geqslant 0} Y_{t}\right\|_{1} \leqslant 3\left\|\sup _{t \geqslant 0} X_{t}\right\|_{1}
$$

and the constant 3 is the best possible.

(ii) If $X$ is a submartingale satisfying $\|X\|_{\infty} \leqslant 1$, then

$$
\left\|\sup _{t \geqslant 0} Y_{t}\right\|_{p} \leqslant 2 \Gamma(p+1)^{1 / p}, \quad 1 \leqslant p<\infty .
$$

The constant $2 \Gamma(p+1)^{1 / p}$ is the best possible.

Mathematics subject classification (2010): Primary: 60G42; secondary: 60G44.

Keywords and phrases: Submartingale, supermartingale, stochastic integral, maximal function.

\section{REFERENCES}

[1] K. Bichteler, Stochastic integration and $L^{p}$-theory of semimartingales, Ann. Probab., 9 (1981), 4989.

[2] D. L. BuRKholder, Explorations in martingale theory and its applications, Ecole d'Éte de Probabilités de Saint-Flour XIX-1989, pp. 1-66, Lecture Notes in Math., 1464, Springer, Berlin, 1991.

[3] D. L. BURKHOLDER, Sharp norm comparison of martingale maximal functions and stochastic integrals, Proceedings of the Norbert Wiener Centenary Congress, 1994 (East Lansing, MI, 1994), pp. 343-358, Proc. Sympos. Appl. Math., 52, Amer. Math. Soc., Providence, RI, 1997.

[4] A. OsȨKows KI, Sharp maximal inequality for stochastic integrals, Proc. Amer. Math. Soc., 136 (2008), 2951-2958.

[5] A. Oş̦KowsKI, Sharp maximal inequality for martingales and stochastic integrals, Electron. Commun. Probab., 14 (2009), 17-30. 